\title{
An Analysis of the Quality of New-Born Health Services in Nampula, Mozambique.
}

Paulo Das Das Neves Pires ( $\Delta$ druidatom@gmail.com )

Lúrio University https://orcid.org/0000-0002-2586-9955

Martins Mupueleque

University Mussa Bin Bique

Jaibo Mucufo

Lúrio University

Ronald Siemens

University of Saskatchewan

Celso Belo

Lúrio University

\section{Research}

Keywords: child health, health services, implementation research, Mozambique, new-born, patient centred care, quality assessment

Posted Date: November 3rd, 2020

DOl: https://doi.org/10.21203/rs.3.rs-99720/v1

License: (c) (i) This work is licensed under a Creative Commons Attribution 4.0 International License.

Read Full License 


\section{Abstract}

\section{Background}

New-born morbidity and mortality are high in Africa, including Mozambique. One important factor to reduce this public health burden is ensuring the frequency and quality of new-born visits, with the availability of efficient, timely, patient centred care. To contribute to the reduction of new-born mortality rate in Nampula, the Lúrio University and the University of Saskatchewan, carried out an implementation research project which included training activities for health professionals in maternal and child health care. We planned a mid-project evaluation, to assess the impact of health professionals training on the quality of services at Marrere Health Centre.

\section{Methods}

Quantitative study, applying two cross-sectional surveys about new-born visits service quality. The first surveys were conducted after two health professionals' training sessions and the other after five more sessions. The samples of carers of infants up to 28 days of age in Marrere Health Centre, were calculated considering the average number of post-partum visits per month (47 in 2018, with a margin of error of $10 \%$ and a confidence interval of $90 \%, 134$ in 2019, with a margin of error of $5 \%$ and a confidence interval of 95\%). The individual surveys used a five-point Likert scale and were entered into REDCap, and analysed to assess frequency, percentage, mean and standard deviation. This research was approved by the bioethics committees at Lúrio University and at the University of Saskatchewan.

\section{Results}

188 child carers were surveyed at Marrere Health Centre, about new-born services quality. Most areas showed no improvement. Positive improvements were a $48 \%$ increase in health professionals encouraging mothers to share any difficulties during the patient encounter, a $31 \%$ increase in encouraging mothers to have a person of their choice to accompany them during labour, suggesting a traditional birth attendant (97\%). Many shortcomings persisted in practices of introducing themselves, communication with patients, privacy, and confidentiality.

\section{Conclusion}

The quality of care at Marrere Health Centre did not improve and health professionals are not practising according to the protocol. Reviewing health professionals learning approach, developing continuous capacity building, would be the next best steps to improve quality of new-born centred care.

\section{Trial registration}

This study was not registered in any data base.

\section{Plain English Summary}


The low number and quality of new-born visits contributes to a high new-born mortality in Mozambique. To approach health problem, we carried out an implementation research project with training activities for health professionals in child health care. We planned a mid-project evaluation, to assess the impact of health professionals training on the quality of services at Marrere Health Centre.

This was a quantitative study, applying two cross-sectional surveys about new-born visits service quality, the first after two health professionals' training sessions, the second after five more sessions. Subjects were carers of infants up to 28 days of age in Marrere Health Centre. This research was approved by the bioethics committees at Lúrio University and at the University of Saskatchewan.

A total of 188 child carers were surveyed at Marrere Health Centre, about new-born services quality. Most areas showed no improvement. Positive improvements were a $31 \%$ increase in health professionals encouraging mothers to have a person of their choice to accompany them during labour, suggesting a traditional birth attendant (97\%).

The quality of care at Marrere Health Centre did not improve. Reviewing health professionals learning approach and developing continuous capacity building, would be the next steps to improve quality of new-born centred care.

\section{Introduction}

Child mortality has its highest incidence in the first year of life and is concentrated in the first month. ${ }^{1}$ Access to and quality of child health services, is essential to achieve Sustainable Development Goal number $3,{ }^{2}$ especially in low-income countries, including reduction of morbidity and mortality rates in children, which in Mozambique is among the highest in Africa and the world. To reduce new-born mortality, the World Health Organisation (WHO), produced in 2016 specific recommendations to the countries. ${ }^{3}$

In 2008, the Mozambican Ministry of Health (MISAU) developed a strategy to accelerate maternal and new-born mortality rates reduction, ${ }^{4}$ but in 2013 , the country registered 27,8 deaths of children less than 28 days of age per 1000 live births, and 88.5 under five years, ${ }^{5}$ with higher mortality risk for those born in Nampula and other northern provinces. ${ }^{6}$ Though child mortality rate decreased in Mozambique in the last three decades, ${ }^{7}$ it is still high (67.3 deaths of children less than one year of age per 1000 live births, 2017). ${ }^{8}$

Among the main causes are the reduced number of qualified health professionals (HPs), lack of equipment and supplies. Additionally, are poor quality of care, deficient referral system, long distances, and lack of transport to access the health unit (HU), poor communication between HPs and the community, and gender issues. These barriers are common to low-and-middle income countries, ${ }^{9}$ mainly in sub-Saharan Africa. ${ }^{10,11}$ 
Although MISAU defined policies to promote child health in primary care at Healthy and at Risk Children Services since $2011,{ }^{12}$ in the last decade, the low quality of maternal and child health $(\mathrm{MCH})$ services in Mozambique, has hardly improved. ${ }^{13}$ An assessment of quality and access to health care, in 195 countries in 2016, placed Mozambique in position 179. ${ }^{14}$

These facts led the Faculty of Health Sciences (FHS) of the Lúrio University (UniLúrio) and the University of Saskatchewan in Canada, to develop an implementation research on $\mathrm{MCH}$, in the administrative post of Natikiri, in Nampula province, Mozambique, called Alert Community to a Prepared Hospital care continuum (ACPH). A baseline study showed a low level of knowledge about sexual and reproductive health (SRH) and rights in the Natikiri population and poor family planning (FP) practice. ${ }^{15}$ Project activities stimulated community participation and SRH and FP education, empowering population health knowledge, attitudes and practice. Another strategy was to improve training of HPs in obstetric emergencies, new born resuscitation, SRH rights, ante-natal consultation and humanization of care in Marrere Health Centre (MHC); some equipment and supplies were also provided, knowing that most newborn deaths can be prevented by effective interventions. ${ }^{16}$

This paper pertains to the results of a planned mid-project evaluation, intended to estimate the impact of HPs training in new-born visits, demonstrated to have a positive impact in the quality of services. ${ }^{17}$

Given the importance of feedback from users to evaluate health services, with regards to the quality of care issues, communication, information, and advice, our implementation research method to collect data, targeted participants citizenship and health empowerment, informing and educating the population.

\section{Method}

\section{Study design}

This was a quantitative pre-post study, applying two cross-sectional surveys on user's opinion about newborn care services quality, the first being conducted after two training sessions on new-born resuscitation (during the 3rd semester of the project, 2018) and the second after five more training sessions, two on new-born resuscitation, two on family-friendly consultation and humanized care, and one in SRH (during the 6th semester of the project, 2019); each training lasted five days, 20 hours in total, given to $30 \mathrm{HPs}$ over the seven modules.

\section{Setting}

Surveys with user groups were privately carried out at the healthy child service, child at risk service and emergency room, in MHC.

\section{Sample}

To calculate representative samples of mothers with children with less than one month of age at MHC, Natikiri administrative post, Nampula, we considered the monthly average number of post-partum visits, 
143 in 2018 , with a margin of error of $10 \%$ and a confidence interval of $90 \%$, attaining 47 women; for the second survey we considered the monthly average number of post-partum visits with children less than one month, 178 in 2019, with a margin of error of 5\% and a confidence interval of 95\%, attaining 122 women, and we added $10 \%$ to compensate registering errors, attaining 134 child' carers.

The two groups are made up of different subjects.

Data collection: participants were submitted to a closed ended survey, previously tested, in Portuguese or Emakhuwa (local language) according to the participant's preference, administered by UniLúrio FHS' students, after being adequately trained and signing ethical and scientific commitment forms. Mothers of new-born children were questioned in private at the MHC facilities, from the 24th to 31st of July 2018 and from the 28th of November to 6th of December 2019. All women were informed they were free to participate voluntarily, or abandon the survey if they wanted, without any consequences in access or quality of care, and signed an informed consent form, including an informed assent term for adolescents under the age of 18 years.

The survey questions (28) with multi option choice, were answered using a 5-point Likert scale (i.e., totally agree, agree, indifferent, disagree, strongly disagree; or, always, often, sometimes, very infrequently, never); and three in depth open questions were also applied by the interviewers. Data collection instruments were evaluated on the quality of completion.

\section{Data analysis}

Quantitative data were introduced into REDCap (Research Eletronic Data Capture) at https://rev.unilurio.ac.mz/umestumafam/redcap, by the same students, accompanied by an FHS lecturer to be consulted as needed. The data were then analysed by a statistics professor to assess frequency, percentage, average and standard deviation.

This study was approved by the Institutional Committee on Bioethics for Health at UniLúrio and the Bioethics Committee at the University of Saskatchewan and followed all Helsinki Declaration (2013) guidelines.

\section{Results}

We surveyed 188 women with children of less than one month of age at the MHC Healthy and at Risk Child services (48 after two HPs trainings, 140 after a total of seven training sessions), with a mean age of 23.38 years (standard deviation 5.8), minimum 14 and maximum 45 years (4.9\% with less than 18 and $4.1 \%$ withy more than 35 years). Concerning education level, $34.3 \%$ are illiterate, $49.3 \%$ completed primary and $15 \%$ secondary, with two (1.4\%) with higher education. The participants' characteristics are detailed in Table I.

The proportions of residence locations changed, with an increase in Natikiri neighbourhood; there was a slight increase in previous pregnancies number and the percentage of home deliveries increased fourfold; 
there was no change in the percentage of women referring miscarriage.

Table 1

Participant characteristics.

\begin{tabular}{|c|c|c|c|c|c|}
\hline & & & $2018(n=48)$ & $2019(n=140)$ & \\
\hline No. & Question & Answer & Post 2 trainings & Post 5 trainings & $\begin{array}{l}\text { Progress } \\
(\%)\end{array}$ \\
\hline \multirow[t]{2}{*}{1} & \multirow[t]{2}{*}{ Residence (\%) } & Natikiri & 83 & 99.3 & 20 \\
\hline & & Other & 17 & 0.7 & -96 \\
\hline \multirow[t]{2}{*}{2} & \multirow{2}{*}{$\begin{array}{l}\text { Number } \\
\text { of previous pregnancies }\end{array}$} & Average (n) & 2.6 & 2.96 & 14 \\
\hline & & $<=3(\%)$ & 75 & 67.9 & -9 \\
\hline 3 & Number of hospital deliveries & Average (n) & 2.3 & 2.28 & 18 \\
\hline 4 & Home births & $(\%)$ & 6 & 24.3 & 305 \\
\hline \multirow[t]{2}{*}{5} & \multirow[t]{2}{*}{ Miscarriages } & No (\%) & 83 & 88.6 & 7 \\
\hline & & Yes (\%) & 17 & 11.4 & -33 \\
\hline
\end{tabular}

The assessment of principles of good care, although confirmed by more than half participants in most questions, show a negative evolution in all areas, including reception, communication with patients, privacy and confidentiality, care during labour and childcare.

Patients felt less (-10\%) welcome at the services, HPs did not greet and offer a seat (-30\%), asked less $(-25 \%)$ the name, if they had any doubts $(-42 \%)$, did not encourage them to ask questions $(-27 \%)$ and state their expectations at the beginning of the consultation (-49\%), and did not explain what they would do, before performing physical examination or other interventions $(-13 \%)$, did not encourage the husband participation caring for the new-born (-28\%).

Some positive points were identified about care during labour: they informed more (48\%) the mothers that they had the right to speak to any HP about their difficulties, they were best (31\%) given the option to have a person of their choice to accompany them during labour, notably a traditional birth attendant (97\%), and were said they could deliver in a position of their choice (89\%).

The last question summarizes the findings of participants perception, asking the children carers how they evaluate their overall experience at MHC: most rated their experience as excellent (34\%) and good (58\%), but the evolution of this service, however, was unfavourable with $10 \%$ increase of unsatisfied users (see Table 2). 
Table 2

Users' opinions about child health services quality at Marrere Health Centre

\begin{tabular}{|c|c|c|c|c|}
\hline & & $2018(n=48)$ & $2019(n=140)$ & \\
\hline Question & Response (\%) & Post 2 trainings & Post 7 trainings & $\begin{array}{l}\text { Change } \\
(\%)\end{array}$ \\
\hline \multirow{4}{*}{$\begin{array}{l}\text { How do you evaluate your } \\
\text { experience at new-born care visit at } \\
\text { Marrere Health Centre? }\end{array}$} & Great & 20.8 & 3.6 & \multirow[t]{2}{*}{-9} \\
\hline & Good & 70.8 & 49.6 & \\
\hline & $\begin{array}{l}\text { Not very } \\
\text { satisfied }\end{array}$ & 2.1 & 12.4 & \multirow[t]{2}{*}{167} \\
\hline & Not satisfied & 4.2 & 4.4 & \\
\hline
\end{tabular}

In answers to the open questions, about what they like in childcare services, the number of carers well satisfied decreased, but in 2019 we see 90 users (64.8\%) liked HP reception and care.

About what they did not like about the service, there was an increase in criticism (19.6\%) in 2019, and 31 users $(22,4 \%)$ refer to HP bad performance including illicit charges, and $5(3,6 \%)$ lack of medicines.

Asked about what they would change to make the service better, we had a reduction of $29.3 \%$ of those who would do nothing, 32 (23.2\%) suggested improving the reception of patients by HPs, better their punctuality and eliminate illicit charges, and $6(4,3 \%)$ to improve medication availability.

\section{Discussion}

Most participants live in the three communal units of Natikiri neighbourhoods (Marrere, Natikiri, Murrapaniua), with an increasing trend over time, and have a low education level, making health preventive attitudes rare in this population.

The mean number of pregnancies per women remains under the national average (5.2), with more than half having three or less pregnancies, probably due to the low group' mean age. Home births increased, or women felt more at ease to reveal it.

Miscarriages (spontaneous and provoked) show no change.

As recommended by the WHO and MISAU, HPs informed delivering women that they had the option to have a person of their choice to accompany them during labour, and this is a low-cost and effective intervention to improve the quality of $\mathrm{MCH}$ care. ${ }^{18}$

In 2019, most children carers in MHC healthy children consultation and children at risk consultation were satisfied with the service, but we can see a more critic evaluation, translating user's health education empowerment. 
Although more than half of the participants refer that HPs are practicing within the principle of good care, they do not systematically proceed according to the MISAU policy and $\mathrm{MCH}$ protocol; they have deficiencies in patients' reception, information, and communication, and in matters of confidentiality. Lack of medicines also remains a challenge, recognised in several low-income countries. ${ }^{19,20}$

HPs respectful and appropriate attitudes towards child carers and their education, are essential to ensure the quality of child health services. ${ }^{21}$ It is recognised that maternal care directly impacts in new-born outcomes.

Our evaluation reveals no significant impact of HPs trainings on child attendance quality at MHC. This finding might be related to the high turnover of $\mathrm{MHC}$ professionals, and their reduction, causing an overload of work to those remaining, associated with a decrease in material resources due to economic shortcomings. On top of this, we verified that one of the seven training modules was not evaluated; in the six evaluated, we had $24 \%$ participants missing in the post-test and mean evaluation of progress was weak (13\%).

However, child health indicators show a general positive quantitative evolution over time, high above the population increase rate (2,8\% per year), despite the lack of $\mathrm{MCH}$ professionals (see Table 3 ).

Nevertheless, nutritional monitoring, a fundamental strategy approaching malnutrition concerning Mozambican children, showed marked decrease.

Table 3

Evolution of child health services indicators at Marrere Health Centre

\begin{tabular}{|c|c|c|c|c|c|c|}
\hline Area & Service & 2016 & 2017 & 2018 & 2019 & $\begin{array}{l}\text { Progress \% } \\
2016-2019\end{array}$ \\
\hline $\begin{array}{l}\text { Post-partum } \\
\text { visit }\end{array}$ & Consultations & 1536 & 1791 & 1711 & 2139 & 39 \\
\hline \multirow[t]{3}{*}{ Child health } & Consultations children $<1$ year & 1132 & 817 & 878 & 4466 & 295 \\
\hline & $\begin{array}{l}\text { Nutritional monitoring children } \\
<1 \text { year }\end{array}$ & 1132 & 817 & 1368 & 422 & -63 \\
\hline & Vaccination BCG & 1412 & 1710 & 1872 & 2040 & 44 \\
\hline
\end{tabular}

Subsequent recommendations for $\mathrm{MHC} \mathrm{MCH}$ professionals, were directly transmitted verbally in followup meetings, and written down and delivered to MHC Director and all HPs.

Facility assessment tools are valuable to assess quality of new-born care and guide priority measures to reduce the burden of child mortality, ${ }^{22}$ but it is necessary to strength health system and data collection 
methods. ${ }^{23}$ We recommend a national MCH HPs training campaign, continuous, ${ }^{24}$ and regular, about skills, values, transforming attitudes, and interpersonal communication. This must be combined with an improvement on HPs working conditions. Those needs have been identified by MISAU since 2009, ${ }^{25}$ and are developed in the Global strategy for women's, children's, and adolescents' health (2016-2039). ${ }^{26}$ Study limitations: as study limitations we point out the location of interviews in MHC, that might have influenced some answers. Another issue is the application of the Likert scale to a population with perceived difficulty in abstract conceptualization, in which the terms totally and partially, or always and most times, may have been not well understood. Another limiting factor in comparing the two studies is the use of a $90 \%$ confidence interval and $10 \%$ margin of error in the first sample, different from the second ( $95 \%$ and $5 \%$ respectively).

\section{Conclusion}

Health systems face new (antimicrobial resistance, climate emergency, Covid-19 pandemic) and old (in Africa, traditional taboos) challenges, and will be forced to develop new intervention methods.

MCH HPs are subjected to a heavy workload, and they do not systematically practice according to protocol, having several shortcomings in patient's reception, information, confidentiality, and communication.

Although most users were satisfied with the care provided, and the child health statistical indicators show improvement in the number of post-partum consultations with children less than one month and less than one year, child mortality incidence remains high.

The Mozambican national health system continually faces challenges, with the scarcity of HPs and low funding, therefore looking for new tools for action. Continuous health sector investment for capacity building, such as HPs training and better working conditions are keys to achieve behaviour change, and better child health services quality. These interventions depend on MISAU innovative and investment to:

1. Ensure the number required and continuity of $\mathrm{MCH} \mathrm{HP}$ at HUs.

2. Better HPs working conditions.

3. Provide the necessary supplies for properly functioning services, including medicines, gloves, masks, and health information and education materials.

4. Promote recurrent trainings of MCH HPs, reinforcement and updating, in new-born care, humanized consultation, and patient centred and family friendly services.

5. A population health information campaign targeting risk perception and preventive attitudes.

\section{Abbreviations}

$\mathrm{ACPH}$ - Alert Community for a Prepared Hospital care continuum

FHS - Faculty of Health Sciences 
FP - Family planning

HP - Health professional

$\mathrm{HU}$ - Health unit

$\mathrm{MCH}$ - Maternal and child health

MHC - Marrere Health Centre

MISAU - Ministry of Health of the Republic of Mozambique

SRH - Sexual and reproductive health

UniLúrio - Lúrio University

WHO - World Health Organization

\section{Declarations}

\section{Ethics approval and consent to participate}

This research was allowed by the FHS of UniLúrio, Nampula Provincial Health Directorate, and approved by Lúrio University Bioethics for Health Committee (02/CBISUL/16), and the University of Saskatchewan Bioethics Committee (15-112).

We followed all Helsinki Declaration (2013) recommendations, all participants were volunteers, anonymity guaranteed, free to desist if uncomfortable without any negative condition, signing or recording an informed declaration consent term. This research had no risk or remuneration to participants. They agreed to give their time and opinion on this topic to benefit the population, contribute to improve public health policy interventions and implementation research, and empower inhabitants with SRH knowledge.

The study did not involve the use of animals.

\section{Consent for publication}

This manuscript does not contain data from any individual person. Not applicable.

The authors declare they have reviewed this manuscript and agree to submit it to BioMed Central Reproductive Health Journal. The FHS at UniLúrio has authorised this publication.

\section{Availability of data and materials}

The datasets used and analysed during the current study are available from the corresponding author on reasonable request. 


\section{Competing interests}

The authors declare they have no competing interests with study design or final report, no financial or personal relationships with other people or organizations that could inappropriately influence this research.

\section{Funding}

The study, including base line and interventions, was carried out with funding from the FHS of UniLúrio, with the aid of a grant from the Innovating for Maternal and Child Health in Africa initiative - a partnership of Global Affairs Canada (GAC), the Canadian Institutes of Health Research (CIHR) and Canada's International Development Research Centre (IDRC), (ALERT COMMUNITY TO PREPARED HOSPITAL CARE CONTINUUM - 108508-001).

\section{Authors contributions}

PP: Study protocol conception and design, data analysis and interpretation, article draft, final approval of the version to be published.

MM: Study protocol design, data treatment, analysis and interpretation, final approval of the version to be published.

JM: Study protocol design, data interpretation, article draft, final approval of the version to be published.

RS: Study protocol conception and design, data interpretation, article draft, final approval of the version to be published.

CB: Study protocol conception and design, data interpretation, article draft, final approval of the version to be published.

\section{Acknowledgements}

Dr. Delmar Mutereda, Asimbawe Kiza and members of the Lúrio University Health Research Students Board.

\section{References}

1. Wallace R, Chaib F, Mayhew M. As taxas de mortalidade infantil caem acentuadamente em mais de metade desde 1990, mas a meta global dos ODM não foi cumprida por uma grande margem. UNICEF. New York. 2015.

2. United Nations. Sustainable development goals. Accessed August 19, 2020. https://www.un.org/sustainabledevelopment/sustainable-development-goals/ 
3. World health statistics overview 2019: monitoring health for the SDGs, sustainable development goals. Geneva: World Health Organization; 2019 (WHO/DAD/2019.1). Licence: CC BY-NC-SA 3.0 IGO.

4. Garrido P. Roteiro para acelerar a redução da Mortalidade materna e neonatal em Moçambique. Ministério da saúde, República de Moçambique. Maputo. 2008.

5. Wang H, Liddell C, Coates $\mathrm{M}$, et al. Global, regional, and national levels of neonatal, infant, andunder5 mortality during 1990-2013: a systematic analysisfor the Global Burden of Disease Study 2013. Lancet 2014; 384: 957-79. Available from: http://dx.doi.org/10.1016/S0140-6736(14)60497-9.

6. Macassa G, Ghilagaber G, Charsmar H, et al. Geographic Differentials in Mortality of Children in Mozambique: Their Implications for Achievement of Millennium Development Goal 4. J HEALTH POPUL NUTR 2012 Sep;30(3):331-345. ISSN 1606-0997 | \$ 5.00+0.20.

7. WHO. Increasing access for child and maternal health care services: the Mozambique experience. World Health Organization. Regional Office for Africa. 2013. ISBN: 978-929023263-6

8. INE. Resultados definitivos censo 2017. Instituto Nacional de Estatística. Maputo. 2019. https://www.ine.gov.mz.

9. Bohren M, Mehrtash H, Fawole B, et al. How women are treated during facility-based childbirth infour countries: a cross-sectional study with labourobservations and community-based surveys.The Lancet Vol 394 November 9, 2019. www.thelancet.com

10. Nkoka O, Chuang T, Chen Y. Association between timingand number of antenatal care visits on uptake of intermittent preventive treatment for malaria during pregnancy among Malawian women. Malar J (2018) 17:211. https://doi.org/10.1186/s12936-018-2360-z.

11. Navale S, Habumugisha L, Amoroso C, et al. Understanding Drivers of Infant Deaths in Rural Rwanda Through Verbal and Social Autopsy: a Mixed Methods Analysis, Annals of Global Health (2017), https://doi.org/doi:10.1016/j.aogh.2017.10.029.

12. Ibrahimo N, Fernandes N, Mikusova S. Normas de atendimento à criança sadia e à criança em risco. Spectrum Graphics Limitada. Maputo. 2011.

13. Chongo L, Amade N, Chavane L, et al. Quality and Humanization of Care Assessment (QHCA). A Study of the Quality of Maternal and New-bornCare Delivered in Mozambique's Model Maternities. Maternal and child Health integrated Program, USAID. Maputo. 2013.

14. Fullman N, Abay S, Cristiana A, et al. Measuring performance on the Healthcare Access andQuality Index for 195 countries and territories and selectedsubnational locations: a systematic analysis from the GlobalBurden of Disease Study 2016. www.thelancet.com Published online May 23, 2018. http://dx.doi.org/10.1016/S0140-6736(18)30994-2.

15. Belo C, Pires P, Josaphat $J$, et al. Maternal and newborn mortality: community opinions on why pregnant women and new-borns are dying in Natikiri, Mozambique. International Journal of Research, Volume 04 Issue 06, May 2017. p-ISSN: 2348-6848, e-ISSN: 2348-795X. https://edupediapublications.or/jornals/index.php/IJR/.

16. You D, Hug L, Ejdemyr S, et al. Levels \& Trends inChildMortality, Report 2015. UN Inter-agency Group forChild Mortality Estimation. United Nations Children's Fund. New York. 2015. 
17. Spitzer F, Steele S, Caloia D, et al. One-year evaluation of the impact of an emergency obstetric and neonatal care training program in Western Kenya. International Journal of Gynecology and Obstetrics (2014), doi: 10.1016/j.ijgo.2014.05.023.

18. WHO. Companion of choice during labour and childbirth for improved quality of care. Human Reproductive Program. World Health Organization. 2020. www.who.int/health-topics/maternalhealth.

19. Hartman S, Loomis E, Russell $\mathrm{H}$, et al. A guide to providing wide-ranging care to newborns. The Journal of Family Practice. April 2018. Vol 67, No 4.

20. Maisonneuve J, Semrau K, Maji P, et al. Effectiveness of a WHO Safe Childbirth Checklist Coachingbased intervention on the availability of Essential Birth Supplies in Uttar Pradesh, India. International Journal for Quality in Health Care, 2018, 1-9. doi: 10.1093/intqhc/mzy086

21. Iliyasu Z, Farouk Z, Lawal A, et al. Care-seeking behavior for neonatal jaundice in rural northern Nigeria. Public Health in Practice 1 (2020) 100006. Elsevier. https://doi.org/10.1016/j.puhip.2020.100006.

22. Brizuela V, Leslie H, Sharma J, et al. Measuring quality of care for all women and newborns: how do we know if we are doing it right? A review of facility assessment tools. Lancet Glob Health 2019; 7: e624-232. Published Online March 18, 2019. http://dx.doi.org/10.1016/S2214-109X(19)30033-6

23. 23. Hailegebriel T, McCord J. Kangaroo Mother Care: Pathways to Sustainability. Save the Children Federation, HNN Healthy Newborn Network 2018.

24. Satveit S. Addressing the unique healthcare needs of women:Opportunity for change exists at the intersection of precisionhealth and learning health systems. Learn Health Sys. 2018;2:e10033.https://doi.org/10.1002//rh2.10033.

25. Chavane L, Dgedge M, Libombo A, et al. Avaliação de necessidades em saúde maternal e neonatal em Moçambique (Parte I). Direcção Nacional de Assistência Médica. Ministério da Saúde, República de Moçambique. Maputo. 2009.

26. Kuruvilla S, Bustreo F, Kuo T, et al. The Global strategy for women's, children's and adolescents' health(2016-2030): a roadmap based on evidence and country experience. Bull World Health Organ 2016;94:398-400. doi: http://dx.doi.org/10.2471/BLT.16.170431

\section{Supplementary Files}

This is a list of supplementary files associated with this preprint. Click to download.

- AQNBHSAnnex1.docx 